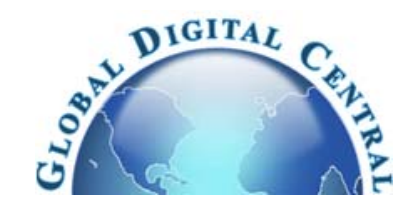

Frontiers in Heat and Mass Transfer

Available at www.ThermalFluidsCentral.org

\title{
AN EXACT SOLUTION ON UNSTEADY MHD VISCOELASTIC FLUID FLOW PAST AN INFINITE VERTICAL PLATE IN THE PRESENCE OF THERMAL RADIATION
}

\author{
E. Kumaresan and A .G. Vijaya Kumar* \\ Department of Mathematics, School of Advanced Sciences, VIT University, Vellore, TN, 632014, India
}

\begin{abstract}
A study has been carried out to analyse an unsteady free convective chemically reacting, MHD Visco-elastic fluid (Walter's liquid-B model) flow past an infinite vertical plate in the presence of thermal radiation with uniform temperature and species diffusion. The dimensionless governing partial differential equations are solved by using Laplace transform technique. The effects of different physical parameters like visco-elastic parameter, chemical reaction parameter, Magnetic field parameter, thermal Grashof number, mass Grashof number and time are discussed by plotting the velocity profiles for both cooling $\left(G_{r}>0, G_{m}>0\right)$ and heating of the plate $\left(G_{r}<0, G_{m}<0\right)$ while the temperature, concentration, Sherwood number and Nusselt number also analysed through graphs. It is observed that increasing viscoelastic and chemical reaction parameter the velocity is increasing for cooling of the plate but the reverse effect to be found in heating of the plate. An increase in the Prandtl number and radiation parameter is found to decrease the velocity and temperature but increasing in Nusselt number.
\end{abstract}

Keywords: Exact solution, Chemical reaction, $M H D$, viscoelastic fluid, Heat and Mass transfer, free convection, thermal radiation

\section{INTRODUCTION}

Several industrial applications involve the flow of non-Newtonian fluids, and thus the flow behavior of such fluids finds a great relevance. Molten metal's, plastic, pulps, emulsions, slurries and raw materials in fluid state are some examples to mention. Non-Newtonian flow also finds practical applications in bio-engineering, wherein blood circulation in human/animal artery is explained by an appropriate visco-elastic fluid model of small elasticity. The study of a visco-elastic pulsatile flow helps in understanding the mechanism of dialysis of blood through an artificial kidney. The constitutive equations of certain class of non-Newtonian fluids with short memories have been proposed by Walters (1964) and Beard and Walters (1964) for elastic-viscous fluid, referred to as Walters Liquid ' $\mathrm{B}$ '. The flow of viscous incompressible fluid past an impulsively started infinite horizontal plate in its own plane was first studied by Stokes (1851). Using this equation several researchers studied the several problems related to the flow of Walter's Liquid B. Bestman (1985) have studied free convection heat transfer to steady radiating Non-Newtonian MHD flow past a vertical porous plate. Jha (1991) has discussed MHD free-convection and mass transfer flow of an elasto-viscous fluid.

Choubey and Yadav (1985) investigated Magneto hydrodynamic flow of a Non-Newtonian fluid past a porous plate. Das and Panda ( 2009) analyzed magneto hydrodynamic steady free convective flow and mass transfer in a rotating elastic-viscous fluid past an infinite vertical porous flat plate with constant suction. Damesh and Shannak (2010) studied visco-elastic fluid flow past an infinite vertical porous plate in the presence of first order chemical reaction. Hameed and Nadeem (2007) discussed Unsteady MHD flow of a Non-Newtonian fluid on a porous plate. Nayak et al. (2013) investigated MHD flow of a viscoelastic fluid along vertical porous surface with chemical reaction.
Rajagopal and Gupta (1984) was investigated an exact solution for the flow of a non-Newtonian fluid past an infinite porous plate.

Samria et al. (1991) studied MHD free convection flow of a viscoelastic fluid past an infinite vertical plate. Rajesh (2011) studied heat source and mass transfer effects on MHD flow of an elasto-viscous fluid through a porous medium. Kumar and Varma (2011) investigated thermal diffusion and radiation effects on unsteady MHD flow past an impulsively started exponentially accelerated vertical plate with variable temperature and variable mass diffusion. Nabil et al. (2012) discussed numerical study of viscous dissipation effects on free convection heat and mass transfer of MHD non-Newtonian fluid flow through a porous medium. Umamaheswar et al. (2013) investigated unsteady MHD free convective visco-elastic fluid flow bounded by an infinite inclined porous plate in the presence of heat source, viscous dissipation and ohmic heat. Attia (2013) studied unsteady flow of a non-Newtonian fluid above a rotating disk with heat transfer. Ramesh et al. (2014) analyzed double diffusive convection in a layer of Maxwell viscoelastic fluid in porous medium in the presence of Soret and Dufour effects.

Rashidi et al. (2014) investigated mixed convective heat transfer for MHD viscoelastic fluid flow over a porous wedge with thermal radiation. Jha et al. (2014) studied influence of Soret effect on MHD mixed convection flow of visco-elastic fluid past a vertical surface with Hall effect. Prakash et al. (2014) investigated the Effects of chemical reaction and radiation absorption on MHD flow of dusty viscoelastic fluid. Ravikumar et al. (2015) discussed theoretical investigation of an unsteady MHD free convection heat and mass transfer flow of a nonNewtonian fluid flow past a permeable moving vertical plate in the presence of thermal diffusion and heat sink. Rushi Kumar et al. (2015) studied thermal diffusion effects on MHD heat and mass transfer flow past a moving vertical plate when the magnetic field relative to the fluid or to the plate. Motivated by these investigations mentioned above, the purpose of the present work is to consider unsteady MHD free convective

*Corresponding Author. Email: vijayakumarag@vit.ac.in. 
chemically reacting viscoelastic fluid flow past an infinite vertical plate with uniform temperature and species concentration.

The governing equations are solved by using Laplace transform technique and the solutions for velocity, temperature, concentration fields as well as for Sherwood number and Nusselt number are derived in terms of exponential and complementary error function analytically without any restriction. The method which we have imposed is very popular and elegant method (this technique used by Stokes in his problem, well knows as 'Stokes first problem' in the literature) to provide the solutions in closer form and on the other hand the exact solutions are needed not only for the technical relevance of the flows but are also significant for a variety of other reasons such as they can be used as a benchmark for numerical solvers and for checking the stability of their solutions. Consequently, the exact solution for the problem under consideration is desirable. However, the principal difficulty in using this method is finding their inverses. Unless the transform is known, an integration must be performed in the complex plane (Bromwich's integral) to find the inverse. Despite the power of complex analysis, this analytical technique often fails and Bromwich's integral must, finally, be integrated numerically.

\section{MATHEMATICAL ANALYSIS}

The unsteady free convection and mass transfer flow of an electrically conducting visco-elastic fluid past an infinite vertical plate in the presence of heat source has been considered. A transverse magnetic field of uniform strength $B_{0}$ is applied normal to the direction of the flow. The induced magnetic field is neglected in comparison to the applied magnetic field as the magnetic Reynolds number of the flow is taken to be very small. The flow is assumed to be in $x^{\prime}$ - direction which is taken along the vertical plate in upward direction against to the gravitational field and the $y^{\prime}$-axis is taken to be normal to the plate. Initially the plate and the surrounding fluid are at the same temperature $T_{\infty}{ }^{\prime}$ with concentration level $C_{\infty}{ }^{\prime}$ at all points in stationary condition. At time $t>0$, the plate is given an impulsive motion with a velocity $u=u_{0}$ in its own plane and all at once the plate temperature and spices concentration are upstretched to $T_{w}^{\prime}$ and $C_{w}^{\prime}$ respectively. For free convection flow, it is also assumed that

- $\quad$ The viscous dissipation is neglected in the energy equation.

- $\quad$ The effects of variation in density ( $\rho$ ) with temperature and species concentration are considered only in the body force term, in accordance with usual Boussinesq's approximation.

- $\quad$ The fluid considered here is gray, absorbing / eliminating radiation but a non-scattering medium.

- Since the flow of the fluid is assumed to be in the direction of $x^{\prime}$ axis, so the physical quantities are functions of the coordinates $y^{\prime}$ and $t^{\prime}$ only.

Then by usual Boussinesq's approximation, the unsteady visco-elastic fluid flow is governed by the following equations.

$$
\begin{aligned}
\frac{\partial u^{\prime}}{\partial t^{\prime}} & =v \frac{\partial^{2} u^{\prime}}{\partial y^{\prime 2}}-\frac{K_{0}}{\rho} \frac{\partial^{3} u^{\prime}}{\partial y^{\prime 2} \partial t^{\prime}}-\frac{\sigma B_{0}^{2} u^{\prime}}{\rho} \\
& +g \beta\left(T^{\prime}-T_{\infty}^{\prime}\right)+g \beta^{*}\left(C^{\prime}-C_{\infty}^{\prime}\right) \\
\rho C_{p} \frac{\partial T^{\prime}}{\partial t^{\prime}}=\kappa \frac{\partial^{2} T^{\prime}}{\partial y^{\prime 2}}-\frac{\partial q_{r}}{\partial y^{\prime}} & \frac{\partial C^{\prime}}{\partial t^{\prime}}=D \frac{\partial^{2} C^{\prime}}{\partial y^{\prime 2}}-K_{r}\left(C^{\prime}-C_{\infty}^{\prime}\right)
\end{aligned}
$$

with the initial boundary conditions: $t^{\prime} \leq 0: \quad u^{\prime}=0, \quad T^{\prime}=T_{\infty}^{\prime}, \quad C^{\prime}=C_{\infty}^{\prime} \quad$ for all $y^{\prime}$

$t^{\prime}>0: u^{\prime}=u_{0}, T^{\prime}=T_{w}^{\prime}, C=C_{w} \quad$ at $y^{\prime}=0$

$u^{\prime}=0, \quad T^{\prime} \rightarrow T_{\infty}^{\prime}, \quad C^{\prime} \rightarrow C_{\infty}^{\prime} \quad$ as $y^{\prime} \rightarrow \infty$,

where $A=\frac{u_{0}^{2}}{v}$. The local radiant for the case of an optically thin gray

gas is expressed by $\frac{\partial q_{r}}{\partial y^{\prime}}=-4 a^{*} \sigma\left(T_{\infty}{ }^{\prime}-T^{\prime 4}\right)$

It is assumed that the temperature differences within the flow are sufficiently small and that $T^{\prime 4}$ may be expressed as a linear function of the temperature .This is obtained by expanding $T^{\prime 4}$ in a Taylor series about $T_{\infty}{ }^{\prime}$ and neglecting the higher order terms, thus, we get

$T^{\prime 4} \cong 4 T_{\infty}^{\prime 3} T^{\prime}-3 T_{\infty}^{\prime 4}$

From equations (5) and (6), equation (2) reduces to

$\rho C_{p} \frac{\partial T^{\prime}}{\partial t^{\prime}}=\kappa \frac{\partial^{2} T^{\prime}}{\partial y^{\prime 2}}+16 a^{*} \sigma T_{\infty}{ }^{\prime 3}\left(T_{\infty}{ }^{\prime}-T^{\prime}\right)$

On introducing the following non-dimensional quantities:

$u=\frac{u^{\prime}}{u_{0}}, t=\frac{t^{\prime} u_{0}^{2}}{v}, y=\frac{y^{\prime} u_{0}}{v}, \theta=\frac{T^{\prime}-T_{\infty}^{\prime}}{T_{w}^{\prime}-T_{\infty}^{\prime}}, G_{r}=\frac{g \beta v\left(T_{w}^{\prime}-T_{\infty}^{\prime}\right)}{u_{0}^{3}}, C=\frac{C-C_{\infty}^{\prime}}{C_{w}^{\prime}-C_{\infty}^{\prime}}, \quad k=\frac{v K_{r}}{u_{0}^{2}}$
$G_{m}=\frac{g \beta^{\prime} v\left(C_{w}^{\prime}-C_{\infty}^{\prime}\right)}{u_{0}^{3}}, \operatorname{Pr}=\frac{\mu C_{p}}{\kappa}, S c=\frac{v}{D}, M=\frac{\sigma B_{0}^{2} v}{\rho u_{0}^{2}}, R=\frac{16 a^{*} v^{2} \sigma T_{\infty}^{\prime 3}}{\kappa u_{0}^{2}}, S=\frac{K_{0}^{2} u_{0}^{2}}{\rho v^{2}}$,

\section{METHOD OF SOLUTION}

In equations (1) to (4), leads to

$$
\begin{aligned}
& \frac{\partial u}{\partial t}=\frac{\partial^{2} u}{\partial y^{2}}-S \frac{\partial^{3} u}{\partial y^{2} \partial t}-M u+G_{r} \theta+G_{m} C \\
& \frac{\partial \theta}{\partial t}=\frac{1}{\operatorname{Pr}} \frac{\partial^{2} \theta}{\partial y^{2}}-\frac{R}{\operatorname{Pr}} \theta \\
& \frac{\partial C}{\partial t}=\frac{1}{S c} \frac{\partial^{2} C}{\partial y^{2}}-k C
\end{aligned}
$$

with the initial and boundary conditions:

$$
\begin{array}{rll}
t \leq 0 ; \quad u=0, \quad \theta=0, \quad C=0 & \text { for all } y \\
t>0 ; & u=1, \quad \theta=1, \quad C=1 & \text { at } y=0 \\
u \rightarrow 0, & \theta \rightarrow 0, C \rightarrow 0 & \text { as } y \rightarrow \infty
\end{array}
$$

All the physical parameters are defined in the nomenclature. For solving the problem, we take Beard and Walters (1964) $u$ in the form $u=u_{0}+S u_{1}$. The solution of equations (9) to (11) under initial and boundary conditions (12) are obtained by usual Laplace transform technique and the solutions for velocity, temperature, concentration as well as the rate of heat transfer and mass transfer are obtained in terms of exponential and complementary error functions as follows:

$$
\begin{aligned}
& \theta=\frac{1}{2}\left[\begin{array}{l}
\exp (y \sqrt{R}) \operatorname{erfc}\left(\frac{y \sqrt{\mathrm{Pr}}}{2 \sqrt{t}}+\sqrt{\frac{R t}{\operatorname{Pr}}}\right) \\
+\exp (-y \sqrt{R}) \operatorname{erfc}\left(\frac{y \sqrt{\mathrm{Pr}}}{2 \sqrt{t}}-\sqrt{\frac{R t}{\operatorname{Pr}}}\right)
\end{array}\right] \\
& C=\frac{1}{2}\left[\begin{array}{l}
\exp (y \sqrt{k S c}) \operatorname{erfc}\left(\frac{y \sqrt{S c}}{2 \sqrt{t}}+\sqrt{k t}\right) \\
+\exp (-y \sqrt{k S c}) \operatorname{erfc}\left(\frac{y \sqrt{S c}}{2 \sqrt{t}}-\sqrt{k t}\right)
\end{array}\right] \\
& u=B_{1}+\frac{G_{r}}{R-P}\left[B_{1}+B_{5}-B_{2}-B_{4}\right]+\frac{G_{m}}{k S c-P}\left[B_{1}+B_{7}-B_{3}-B_{6}\right] \\
& +\frac{S G_{r} R}{(\operatorname{Pr}-1)^{2}}\left[\exp (-b t)\left(B_{8}-B_{10}\right)\right]-\frac{S G_{m} y}{2(S c-1)}\left[B_{13}+B_{15}\right] \\
& +\frac{S G_{r} \operatorname{Pr}}{(\operatorname{Pr}-1)^{2}}\left[b \exp (-b t)\left(B_{10}-B_{8}\right)+\left(B_{2}-B_{5}\right)\right]-\frac{S G_{r} y}{2(\operatorname{Pr}-1)}\left[B_{13}+B_{14}\right] \\
& +\frac{S G_{m} k S c}{(S c-1)^{2}}\left[\exp (-c t)\left(B_{9}-B_{11}\right)\right]-\frac{S y}{2}\left[B_{12}\right]
\end{aligned}
$$


$+\frac{S G_{m} S c}{(S c-1)^{2}}\left[c \exp (-c t)\left(B_{11}-B_{9}\right)+\left(B_{3}-B_{7}\right)\right]$

where $b=\frac{R-M}{\operatorname{Pr}-1}, c=\frac{k S c-M}{S c-1}$

$B_{1}=\frac{1}{2}\left[\begin{array}{l}\exp (y \sqrt{M}) \operatorname{erfc}\left(\frac{y}{2 \sqrt{t}}+\sqrt{M t}\right) \\ +\exp (-y \sqrt{M}) \operatorname{erfc}\left(\frac{y}{2 \sqrt{t}}-\sqrt{M t}\right)\end{array}\right]$

$B_{2}=\frac{\exp (-b t)}{2}\left[\begin{array}{l}\exp (y \sqrt{M-b}) \operatorname{erfc}\left(\frac{y}{2 \sqrt{t}}+\sqrt{(M-b) t}\right) \\ +\exp (-y \sqrt{M-b}) \operatorname{erfc}\left(\frac{y}{2 \sqrt{t}}-\sqrt{(M-b) t}\right)\end{array}\right]$

$B_{3}=\frac{\exp (-c t)}{2}\left[\begin{array}{l}\exp (y \sqrt{M-c}) \operatorname{erfc}\left(\frac{y}{2 \sqrt{t}}+\sqrt{(M-c) t}\right) \\ +\exp (-y \sqrt{M-c}) \operatorname{erfc}\left(\frac{y}{2 \sqrt{t}}-\sqrt{(M-c) t}\right)\end{array}\right]$

$B_{4}=\frac{1}{2}\left[\begin{array}{l}\exp (y \sqrt{R}) \operatorname{erfc}\left(\frac{y \sqrt{\mathrm{Pr}}}{2 \sqrt{t}}+\sqrt{\frac{R t}{\mathrm{Pr}}}\right) \\ +\exp (-y \sqrt{R}) \operatorname{erfc}\left(\frac{y \sqrt{\mathrm{Pr}}}{2 \sqrt{t}}-\sqrt{\frac{R t}{\mathrm{Pr}}}\right)\end{array}\right]$

$B_{5}=\frac{\exp (-b t)}{2}\left[\begin{array}{l}\exp (y \sqrt{R-b \operatorname{Pr}}) \operatorname{erfc}\left(\frac{y \sqrt{\operatorname{Pr}}}{2 \sqrt{t}}+\sqrt{\frac{R t}{\operatorname{Pr}}-b t}\right) \\ +\exp (-y \sqrt{R-b \operatorname{Pr}}) \operatorname{erfc}\left(\frac{y \sqrt{\operatorname{Pr}}}{2 \sqrt{t}}-\sqrt{\frac{R t}{\operatorname{Pr}}-b t}\right)\end{array}\right]$

$B_{6}=\frac{1}{2}\left[\begin{array}{l}\exp (y \sqrt{k S c}) \operatorname{erfc}\left(\frac{y \sqrt{S c}}{2 \sqrt{t}}+\sqrt{k t}\right) \\ +\exp (-y \sqrt{k S c}) \operatorname{erfc}\left(\frac{y \sqrt{S c}}{2 \sqrt{t}}-\sqrt{k t}\right)\end{array}\right]$

$B_{7}=\frac{\exp (-c t)}{2}\left[\begin{array}{l}\exp (y \sqrt{(k-c) S c}) \operatorname{erfc}\left(\frac{y \sqrt{S c}}{2 \sqrt{t}}+\sqrt{(k-c) t}\right) \\ +\exp (-y \sqrt{(k-c) S c}) \operatorname{erfc}\left(\frac{y \sqrt{S c}}{2 \sqrt{t}}-\sqrt{(k-c) t}\right)\end{array}\right]$

$B_{8}=\left[\begin{array}{l}\left(\frac{t}{2}+\frac{y}{4 \sqrt{M-b}}\right) \exp (y \sqrt{M-b}) \operatorname{erfc}\left(\frac{y}{2 \sqrt{t}}+\sqrt{(M-b) t}\right) \\ +\left(\frac{t}{2}-\frac{y}{4 \sqrt{M-b}}\right) \exp (-y \sqrt{M-b}) \operatorname{erfc}\left(\frac{y}{2 \sqrt{t}}-\sqrt{(M-b) t}\right)\end{array}\right]$

$B_{9}=\left[\begin{array}{l}\left(\frac{t}{2}+\frac{y}{4 \sqrt{M-c}}\right) \exp (y \sqrt{M-c}) \operatorname{erfc}\left(\frac{y}{2 \sqrt{t}}+\sqrt{(M-c) t}\right) \\ +\left(\frac{t}{2}-\frac{y}{4 \sqrt{M-c}}\right) \exp (-y \sqrt{M-c}) \operatorname{erfc}\left(\frac{y}{2 \sqrt{t}}-\sqrt{(M-c) t}\right)\end{array}\right]$

$B_{10}=\left[\begin{array}{l}\left(\frac{t}{2}+\frac{y \operatorname{Pr}}{4 \sqrt{R-b \operatorname{Pr}}}\right) \exp (y \sqrt{R-b \operatorname{Pr}}) \operatorname{erfc}\left(\frac{y \sqrt{\operatorname{Pr}}}{2 \sqrt{t}}+\sqrt{\frac{R t}{\operatorname{Pr}}-b t}\right) \\ +\left(\frac{t}{2}-\frac{y \operatorname{Pr}}{4 \sqrt{R-b \operatorname{Pr}}}\right) \exp (-y \sqrt{R-b \operatorname{Pr}}) \operatorname{erfc}\left(\frac{y \sqrt{\operatorname{Pr}}}{2 \sqrt{t}}-\sqrt{\frac{R t}{\operatorname{Pr}}-b t}\right)\end{array}\right]$

$B_{11}=\left[\begin{array}{l}\left(\frac{t}{2}+\frac{y \sqrt{S c}}{4 \sqrt{k-c}}\right) \exp (y \sqrt{(k-c) S c}) \operatorname{erfc}\left(\frac{y \sqrt{S c}}{2 \sqrt{t}}+\sqrt{(k-c) t}\right) \\ +\left(\frac{t}{2}-\frac{y \sqrt{S c}}{4 \sqrt{k-c}}\right) \exp (-y \sqrt{(k-c) S c}) \operatorname{erfc}\left(\frac{y \sqrt{S c}}{2 \sqrt{t}}-\sqrt{(k-c) t}\right)\end{array}\right]$

$B_{12}=\left(\frac{y^{3}-2 t}{4 t^{2} \sqrt{\pi t}}\right) \exp \left[-\left(\frac{y^{2}}{4 t}+M t\right)\right]$

$B_{13}=\frac{1}{\sqrt{\pi t}} \exp \left[-\left(\frac{y^{2}}{4 t}+M t\right)\right]$

$$
\begin{aligned}
& B_{14}=\frac{\exp (-b t) \sqrt{M-b}}{2}\left[\begin{array}{l}
\exp (-y \sqrt{M-b}) \operatorname{erfc}\left(\frac{y}{2 \sqrt{t}}-\sqrt{(M-b) t}\right) \\
-\exp (y \sqrt{M-b}) \operatorname{erfc}\left(\frac{y}{2 \sqrt{t}}+\sqrt{(M-b) t}\right)
\end{array}\right] \\
& B_{15}=\frac{\exp (-c t) \sqrt{M-c}}{2}\left[\begin{array}{l}
\exp (-y \sqrt{M-c}) \operatorname{erfc}\left(\frac{y}{2 \sqrt{t}}-\sqrt{(M-c) t}\right) \\
-\exp (y \sqrt{M-c}) \operatorname{erfc}\left(\frac{y}{2 \sqrt{t}}+\sqrt{(M-c) t}\right)
\end{array}\right]
\end{aligned}
$$

\section{The Rate of Heat Transfer}

From temperature filed, now we study the rate of heat transfer in terms of Nusselt number which is given in non-dimensional form as:

$$
N u=-\left[\frac{d \theta}{d y}\right]_{y=0}
$$

follows:

From equations (13) and (16), we get Nusselt number as

$$
N u=\sqrt{\frac{\operatorname{Pr}}{\pi t}} \exp \left(-\frac{R t}{\operatorname{Pr}}\right)+\sqrt{R} \operatorname{erf}\left(\sqrt{\frac{R t}{\operatorname{Pr}}}\right)
$$

\section{The Rate of Mass Transfer}

From concentration filed, now we study the rate of mass transfer which is given in non-dimensional form as:

$$
S h=-\left(\frac{d C}{d y}\right)_{y=0}
$$

From equations (14) and (18), we get Sherwood number as follows:

$S h=\sqrt{\frac{S c}{\pi t}} \exp (-k t)+\sqrt{k S c} \operatorname{erf}(\sqrt{k t})$

\section{RESULTS AND DISCUSSION}

An exact solution to the problem of unsteady MHD free convective chemically reacting visco-elastic fluid flow past a moving vertical plate in the presence of thermal radiation have been presented in the preceding section. In order to get the physical insight into the problem, the numerical values of the velocity field is computed for different values of the system parameters such as $M$ (Magnetic parameter), $S$ (Viscoelastic parameter), $G_{m}$ (mass Grashof number), $G_{r}$ (Thermal Grashof number), $R$ (Radiation parameter), Pr (Prandtl number), $k$ (chemical Reaction parameter), $t$ (time) respectively. Throughout the computations we employ $G_{r}=10, G_{m}=5, k=5, P r=7, S c=0.78, R=4, M=3, S=0.5 t=0.4$. Figure 1 reveals that the velocity variations with viscoelastic parameter $S$ in case of cooling and heating of the plate at time $t=0.4$. It is observed that the elasticity of the fluid increases and then decreases in case of cooling of the plate, whereas it decreases in the case of heating of the plate, finally takes asymptotic values 2.5 for both the cases. It may be concluded that the energy due elastic property of the fluid increases the velocity and then gets dissipated.

Figure 2 in case of cooling and heating of the plate. It is observed that the velocity of the fluid decreases with the increase of the magnetic parameter values for cooling of the plate at time 0.4. As expected, the velocity decreases with an increase in the magnetic parameter. It is because the application of the transverse magnetic field will result in a resistive type force (Lorentz force) similar to the drag force which tends to resist the fluid flow and thus reducing its velocity. Also, the boundary layer thickness decreases with an increase in the magnetic parameter. We also see that velocity profiles decrease with the increase of the magnetic effect indicating that the magnetic field tends to retard the motion of the fluid. The magnetic field may control the flow characteristics. The reverse phenomenon is found in the case of heating of the plate. The effect of the chemical reaction parameter $(k)$ has shown Figure 3 in the case cooling and heating of the plate. As expected, the presence of the 
chemical reaction significantly affects both profiles. It should be mentioned that the case studied relates to a destructive chemical reaction. In fact, as the chemical reaction parameter increases, a considerable reduction in the velocity occurs, and the presence of the peak indicates that the maximum velocity takes place in the fluid body close to the surface, but not at the surface itself. It is evident that an increase in this parameter significantly alters the concentration boundary-layer thickness but does not change the momentum one.

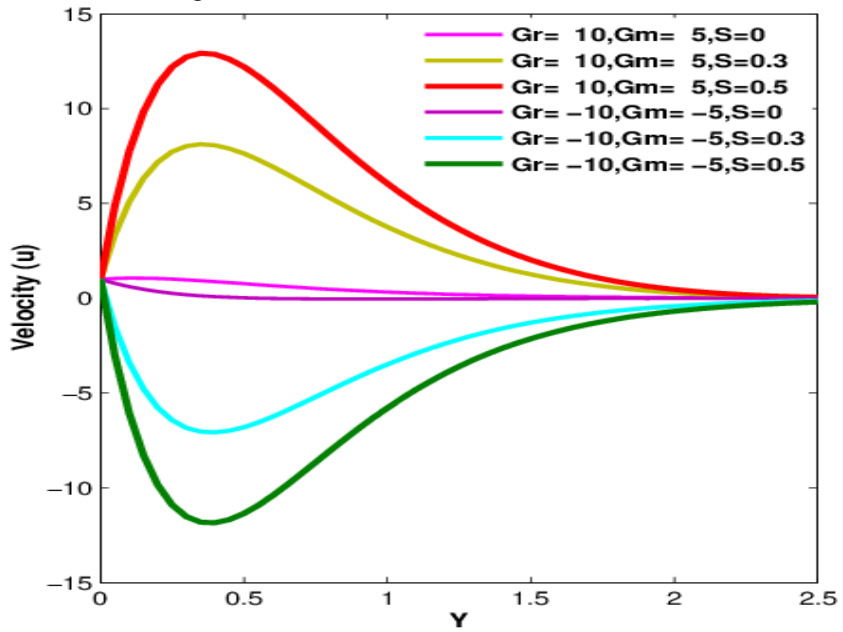

Fig. 1 Velocity profiles for different values of $S$

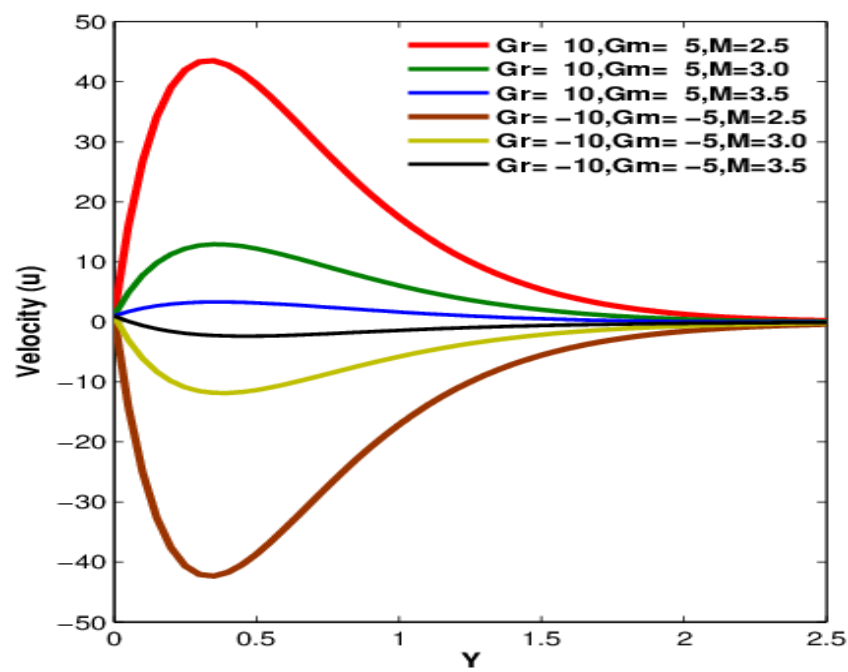

Fig. 2 Velocity profiles for different values of $M$

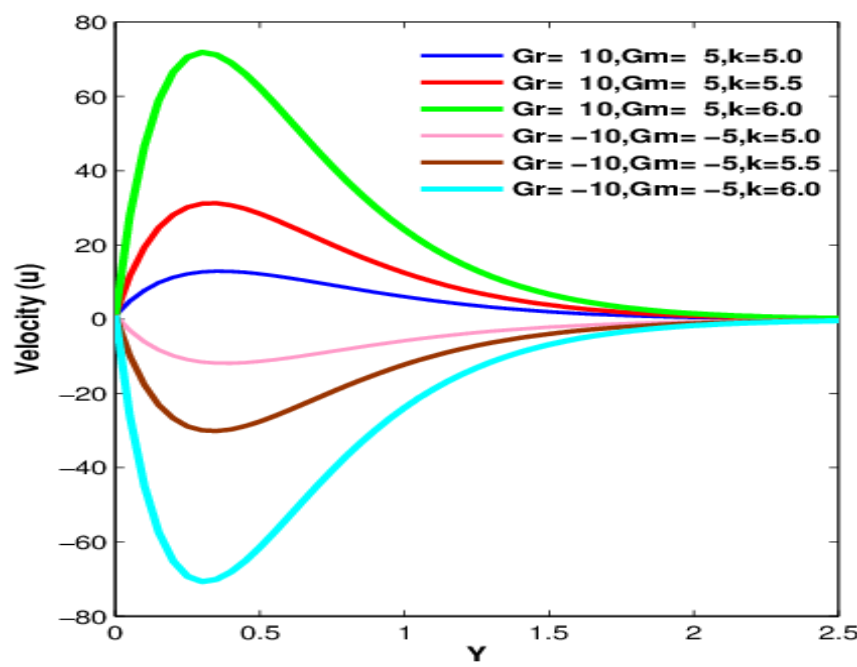

Fig. 3 Velocity profiles for different values of $k$
Figure 4 represents the velocity profiles for different values of $t$ (time) in cases of cooling and heating of the plate respectively. From these figures, in the case of cooling of the plate, the velocity is found to increase with an increase in time t. But the reverse effect is observed in the case of heating of the plate.

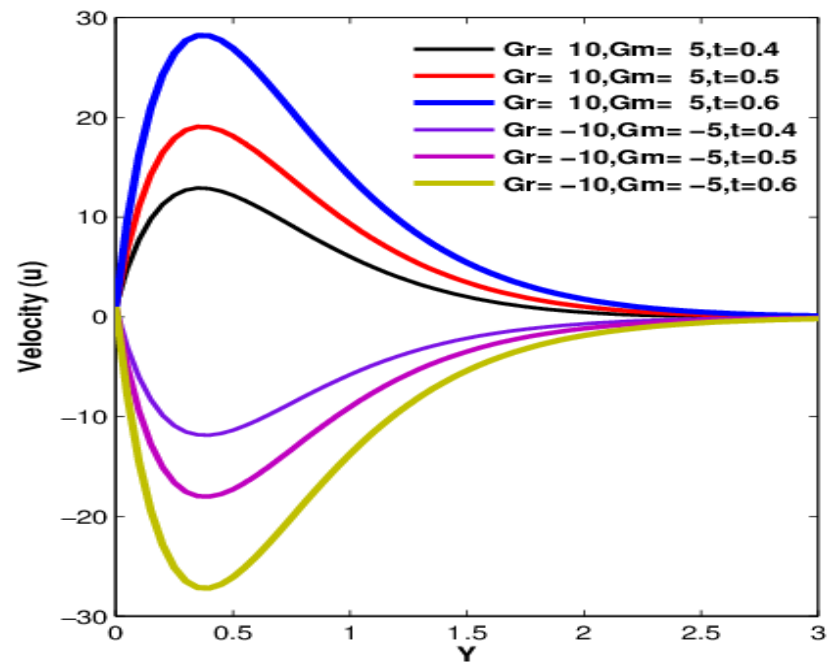

Fig. 4 Velocity profiles for different values of $t$

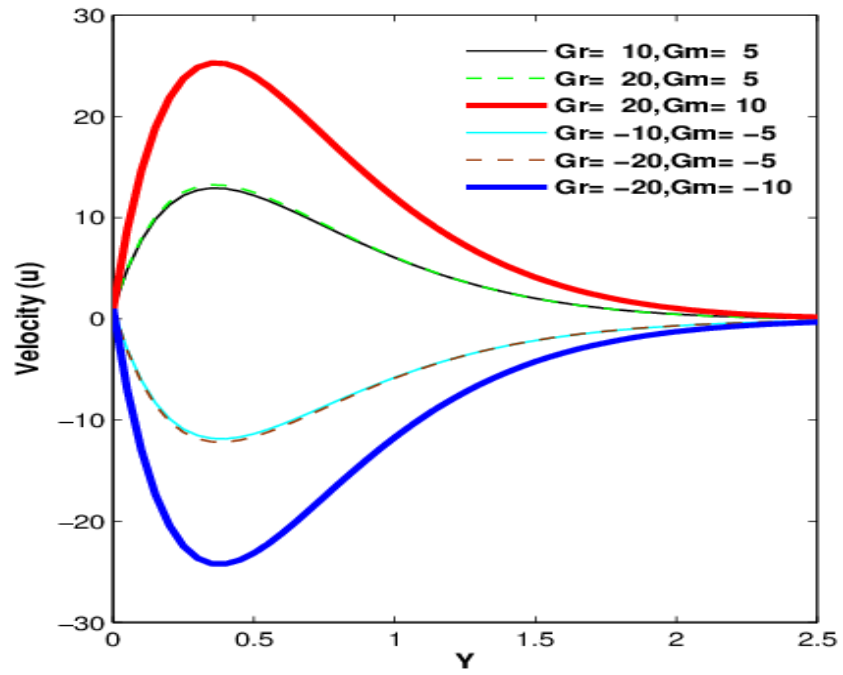

Fig. 5 Velocity profiles for different values of $G r$ and $G m$

Figure 5 shows the effects of thermal Grashof number Gr and mass Grashof number Gm on the velocity profiles. From this figure it is found that the velocity increases in case of cooling of the plate. It is because that increase in the values of thermal Grashof number and mass Grashof number has the tendency to increase the thermal and mass buoyancy effect. This gives rise to an increase in the induced flow transport and a reverse effect is identified in case of heating of the plate. Figure 5 show the effects of thermal Grashof number $\mathrm{Gr}$ and mass Grashof number $\mathrm{Gm}$ on the velocity profiles. From this figure it is found that the velocity increases in case of cooling of the plate. It is because that increase in the values of thermal Grashof number and mass Grashof number has the tendency to increase the thermal and mass buoyancy effect. This gives rise to an increase in the induced flow transport and a reverse effect is identified in case of heating of the plate. The temperature of the flow field is mainly affected by the flow parameters, namely, radiation parameter $(R)$ and time $(t)$. From Figure 6 it is observed that as radiation parameter $\mathrm{R}$ increases, the temperature of the flow field decreases at all the points in flow region. Hence, it is observed that the temperature for conducting air $(\operatorname{Pr}=0.71)$ is higher than that of water $(\operatorname{Pr}=7.0)$ this is because of the fact that thermal conductivity of the fluid decreases with 
increasing values of $\mathrm{Pr}$ resulting decrease in thermal boundary layer thickness. Therefore, using radiation we can control temperature distribution and flow transport. It is also seen that temperature increases as time $t$ increases, and it leads to zero as it moves away from the plate

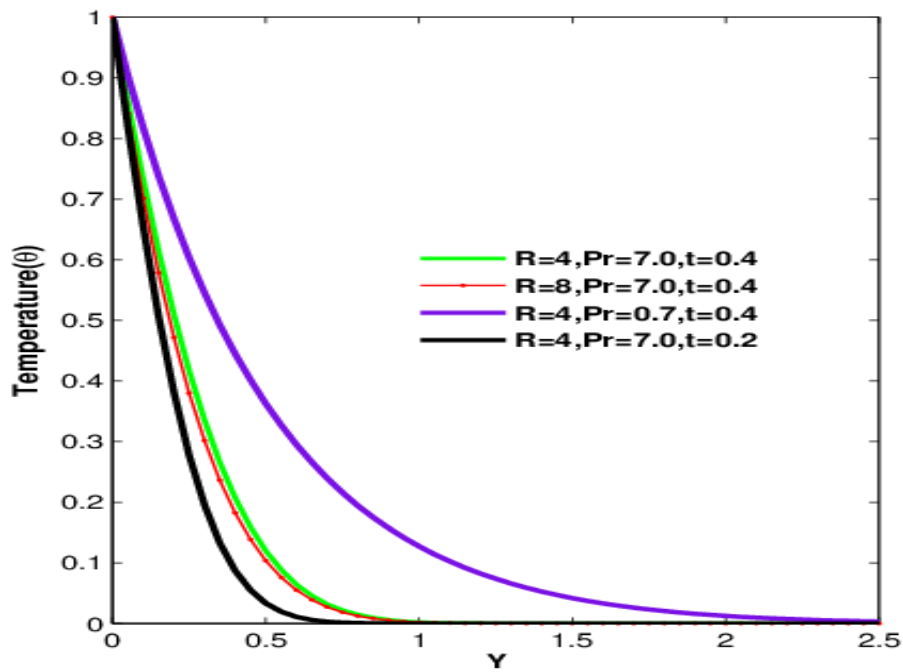

Fig. 6 Temperature profiles for different values of $\mathrm{R}$ and $\mathrm{Pr}$

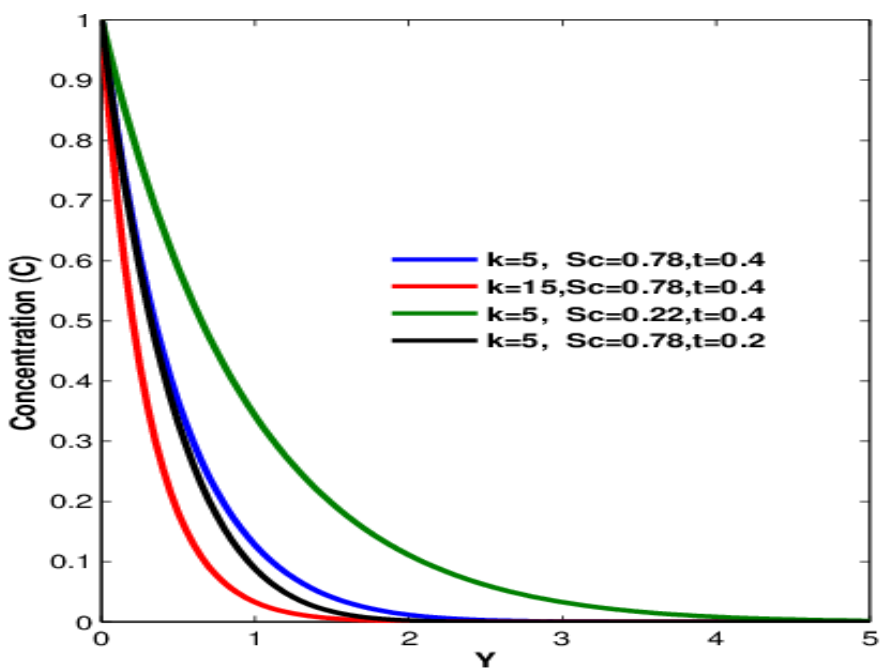

Fig. 7 Concentration profiles for different values of $k$ and Sc

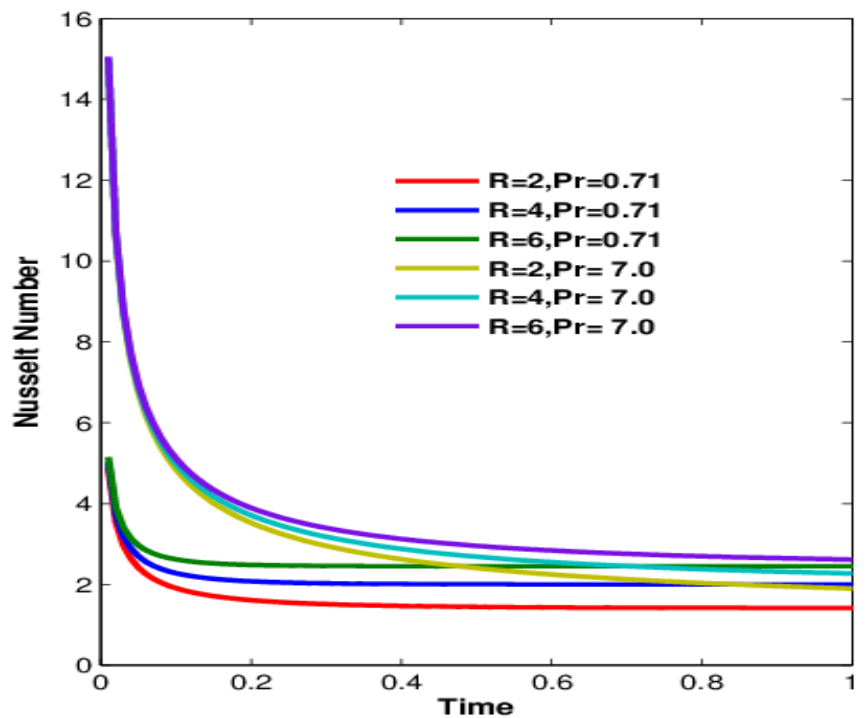

Fig. 8 Nusselt number variation of R for water and air

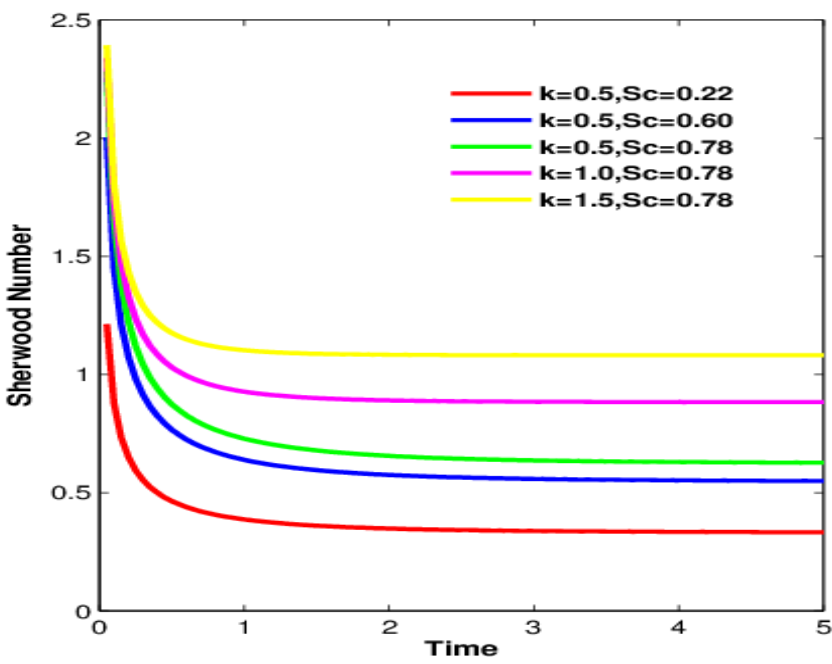

Fig. 9 Sherwood number variation of $k$ and Sc with time

The effect of concentration profiles for different values of chemical reaction parameter, Schmidt number and time are illustrated in Figure 7 it is found that the concentration decreases as chemical reaction parameter or Schmidt number increases while it increases with increasing values of time $t$. It is obviously seen that from Figure (8) the rate of heat transfer is measured in terms of Nusselt number, is observed to increase with an increase in $R$ for both water $(\mathrm{Pr}=7.0)$ and conducting air $(\mathrm{Pr}=$ 0.71). It is also observed that the rate of heat transfer in water is more than in air, it is due to the fact that the smaller values of $\mathrm{Pr}$ are equivalent to increasing the thermal conductivities and therefore heat is able to diffuse more away from surface more rapidly than greater values of $\mathrm{Pr}$, hence there is a reduction in heat transfer coefficient. Finally, from Figure (9) it is noticed that Sherwood number increases as Schmidt parameter or chemical reaction parameter increases.

Table 1 -2 illustrate that the effects of radiation parameter on the velocity for both cooling and heating case. It can be seen that increasing radiation parameter the velocity is decelerated for cooling case whereas in heating case the reverse effect is identified. The effects of Prandtl number on the velocity field is demonstrated through Tables 3 and 4 for both cases of cooling and heating o of the surface. It can be seen that the velocity decelerates for increasing Prandtl number for cooling circumstances and in contrast the velocity accelerates for heating instance.

Table: 1 Velocity for different $R$ value for cooling case

\begin{tabular}{|l|c|c|c|c|}
\hline \multirow{2}{*}{} & $\mathrm{y}$ & $\mathrm{R}=4$ & $\mathrm{R}=6$ & $\mathrm{R}=8$ \\
\cline { 2 - 5 } & 0 & 1.0000 & 1.0000 & 1.0000 \\
& 0.2 & 11.2755 & 11.2587 & 11.2456 \\
& 0.4 & 12.8616 & 12.8428 & 12.8269 \\
Cooling & 0.6 & 11.1233 & 11.1107 & 11.0994 \\
of the & 0.8 & 8.5143 & 8.5088 & 8.5035 \\
plate & 1.0 & 6.0490 & 6.0480 & 6.0469 \\
& 1.2 & 4.0599 & 4.0610 & 4.0618 \\
& 1.4 & 2.5863 & 2.5878 & 2.5891 \\
& 1.6 & 1.5592 & 1.5606 & 1.5618 \\
& 1.8 & 0.8820 & 0.8830 & 0.8839 \\
& 2.0 & 0.4609 & 0.4615 & 0.4621 \\
\hline
\end{tabular}

Table: 2 Velocity for different $R$ value in heating case

\begin{tabular}{|l|c|c|c|c|}
\hline \multirow{2}{*}{} & $\mathrm{y}$ & $\mathrm{R}=4$ & $\mathrm{R}=6$ & $\mathrm{R}=8$ \\
\cline { 2 - 5 } & 0 & 1.0000 & 1.0000 & 1.0000 \\
& 0.2 & -9.8516 & -9.8348 & -9.8217 \\
Heating & 0.4 & -11.8489 & -11.8301 & -11.8142 \\
of the & 0.6 & -10.4168 & -10.4042 & -10.3929 \\
plate & 0.8 & -8.0517 & -8.0461 & -8.0409 \\
& 1.0 & -5.7923 & -5.7913 & -5.7902 \\
& 1.2 & -3.9786 & -3.9796 & -3.9804 \\
& 1.4 & -2.6459 & -2.6474 & -2.6488 \\
& 1.6 & -1.7185 & -1.7199 & -1.7211 \\
& 1.8 & -1.0962 & -1.0972 & -1.0981 \\
& 2.0 & -0.6887 & -0.6893 & -0.6899 \\
\hline
\end{tabular}


Table: 3 Velocity for different $\operatorname{Pr}$ value in cooling case

\begin{tabular}{|l|c|c|c|}
\hline \multirow{4}{*}{ Cooling of } & $\mathrm{y}$ & $\operatorname{Pr}=0.1$ & $\operatorname{Pr}=0.71$ \\
\cline { 2 - 4 } & 0 & 1.0000 & 1.0000 \\
the plate & 0.2 & 11.2184 & 3.1840 \\
& 0.4 & 12.8812 & 3.5555 \\
& 0.6 & 11.2640 & 3.2660 \\
& 0.8 & 8.7486 & 2.7662 \\
& 1.0 & 6.3190 & 2.2208 \\
& 1.2 & 4.3142 & 1.6936 \\
& 1.4 & 2.7943 & 1.2183 \\
& 1.6 & 1.7106 & 0.8179 \\
& 1.8 & 0.9800 & 0.5051 \\
& 2.0 & 0.5158 & 0.2808 \\
\hline
\end{tabular}

Table: 4 Velocity for different $\operatorname{Pr}$ value in heating case

\begin{tabular}{|l|c|c|c|}
\hline & $\mathrm{y}$ & $\operatorname{Pr}=0.1$ & $\operatorname{Pr}=0.71$ \\
\cline { 2 - 4 } & 0 & 1.0000 & 1.0000 \\
& 0.2 & -9.7945 & -1.7601 \\
Heating of & 0.4 & -11.8685 & -2.5428 \\
the plate & 0.6 & -10.5574 & -2.5595 \\
& 0.8 & -8.2859 & -2.3036 \\
& 1.0 & -6.0623 & -1.9641 \\
& 1.2 & -4.2328 & -1.6122 \\
& 1.4 & -2.8540 & -1.2780 \\
& 1.6 & -1.8699 & -0.9772 \\
& 1.8 & -1.1942 & -0.7193 \\
& 2.0 & -0.7436 & -0.5086 \\
\hline
\end{tabular}

\section{CONCLUSIONS}

We have examined the unsteady free convective chemically reacting, MHD visco-elastic fluid (Walter's liquid-B model) flow past an infinite vertical plate with uniform temperature and also with uniform mass diffusion in the presence of thermal radiation. The dimensionless governing partial differential equations are solved by usual Laplace transform technique, we can conclude the following:

1. The fluid velocity increases with increasing parameters $S, k$, $t, G_{r}$ and $G_{m}$ for cooling of the plate whereas the reverse effect is found in the case of heating of the plate.

2. The fluid velocity decreases with increasing values of the parameters $M, R$ and Prfor cooling of the plate, for heating of the plate the velocity increases.

3. The fluid temperature decreases with increasing values of $R$ (radiation parameter) or $\operatorname{Pr}$ (Prandtl number) while it increases with $t$ (time).

4. The fluid concentration decreases with increase in $k$ (chemical reaction parameter) and $S c$ (Schmidt number) while it increases with $t$ (time).

5. The Nusselt number is observed to increase with an increment $R$ (radiation parameter) or $\operatorname{Pr}$ (Prandtl number).

6. The Sherwood number is found that increasing with the increase of Sc (Schmidt parameter) or $k$ (chemical reaction parameter)

\section{ACKNOWLEDGEMENTS}

The authors are thankful to the reviewers for their suggestions, which have significantly improved the quality of our research paper.

\section{NOMENCLATURE}

$C_{\infty}{ }^{\prime}$ Concentration of the fluid far away from the plate

$C_{w}{ }^{\prime}$ Concentration near to the plate

A Constant

$y^{\prime} \quad$ Coordinate axis normal to the plate

$C$ Dimensionless concentration

$y$ Dimensionless coordinate axis normal to the plate

$u \quad$ Dimensionless velocity
$B_{0} \quad$ External magnetic field

$G_{m} \quad$ Mass Grashof number

$G_{r} \quad$ Thermal Grashof number

Pr Prandtl number

Sc Schmidt number

$C^{\prime}$ Species concentration in the fluid

$C_{p}$ Specific heat at constant pressure

$C_{s} \quad$ Concentration susceptibility

$T_{\infty}{ }^{\prime}$ Temperature of the fluid far away from the plate

$T_{w}{ }^{\prime}$ Temperature of the plate

$T^{\prime} \quad$ Temperature of the fluid near the plate

$\kappa$ Thermal conductivity of the fluid

$t^{\prime}$ Time

$u^{\prime} \quad$ Velocity of the fluid in the $\chi^{\prime}$ - direction

$u_{0} \quad$ Velocity of the plate

$D$ Chemical molecular diffusivity

$g$ Acceleration due to gravity

M Magnetic field parameter

$R \quad$ Radiation parameter

$S \quad$ Visco-elastic parameter

$t$ Dimensional time

$K_{r}$ Dimensional chemically reaction parameter

$k \quad$ Dimensionless chemically reaction parameter

$\mu \quad$ Coefficient of viscosity

erfc Complementary error function

erf Error function

$\rho \quad$ Density of the fluid

$\theta \quad$ Dimensionless temperature

$\sigma \quad$ Electric conductivity

$v \quad$ Kinematic viscosity

$\alpha \quad$ Thermal diffusivity

$\beta^{*}$ Volumetric coefficient of expansion with concentration

$\beta \quad$ Volumetric coefficient of thermal expansion

$W \quad$ Conditions on the wall

$\infty$ Free stream conditions

\section{REFERENCES}

Walters, K., 1964, "Second-order Effects in Elasticity, Plasticity and Fluid Dynamics,” Pergamon, New York, p. 507.

Beard, D., Walters, K., 1964, "Elastico-viscous boundary-layer flows. Part I. Two Dimensional Flow near a Stagnation Point," Proc. Camb.Phil. Soc. 60, 667.

https://doi.org/10.1017/s0305004100038147

Stokes, G.G., 1851, "Flow of Viscous Incompressible Fluid Past an Impulsively Started Infinite Horizontal Plate," Cambridge Phil. Trans. (9)8.

Bestman, A.R., 1985, "Free Convection Heat Transfer to Steady Radiating Non-Newtonian MHD Flow Past a Vertical Porous Plate," Int.J. Numerical Methods in engineering. 21, 899-908. https://doi.org/10.1002/nme.1620210509

Basant Kumar Jha., 1991, "MHD Free-Convection and Mass Transfer Flow of an Elasto-Viscous Fluid," Astrophysics and Space Science. 185(1), 129-135.

https://doi.org/10.1007/bf00642711 
Chowdhury, M. K., Islam, M. N., 2000, "MHD Free Convection Flow of Visco-Elastic Fluid Past an Infinite Vertical Porous Plate,” Heat and Mass transfer.36(5), 439 - 447.

https://doi.org/10.1007/s002310000103

Choubey, K.R., Yadav, R.R., 1985, "Magneto Hydrodynamic Flow of a Non-Newtonian Fluid Past a Porous Plate," Astrophysics and Space Science.115, 345-351.

https://doi.org/10.1007/bf00653811

Das, S.S., Panda, J.P., 2009, “ Magneto Hydrodynamic Steady Free Convective Flow and Mass Transfer in a Rotating Elastic-Viscous Fluid Past an Infinite Vertical Porous Flat Plate With Constant Suction," AMSE Mod meascont B. 78, 1-19.

Hameed, M., Nadeem., 2007, "Unsteady MHD Flow of a NonNewtonian Fluid on a Porous Plate,"J.math.Anal. 325, 724-733. https://doi.org/10.1016/j.jmaa.2006.02.002

Damesh, R.A., Shannak, B.A., 2010, "Visco-Elastic Fluid Flow Past an PInfinite Vertical Porous plate in The Presence of First Order Chemical Reaction," App. math. Mech. 31, 955-962. https://doi.org/10.1007/s10483-010-1330-z

Rajesh, V., 2011, "Heat Source and Mass Transfer Effects on MHD Flow of an Elasto-Viscous Fluid Through a Porous Medium," Annals of faculty engineering Hunedoara. Int. J of engineering. 1584-2665.

Kumar,A.G.V., and Vijaya Kumar Varma,S., 2011, “Thermal Diffusion and Radiation Effects on Unsteady MHD Flow Past an Impulsively Started Exponentially Accelerated Vertical Plate with Variable Temperature and Variable Mass Diffusion," Int. J. of Applied Mathematical Analysis and Applications, Vol.6, No.1- 2, pp.169-192.

Nayak, A., Dash, G.C., Panda., 2013, “ MHD Flow of a Visco-Elastic Fluid along Vertical Porous Surface With Chemical Reaction,”A.Phys.Sci. 83(2), 153-161.

https://doi.org/10.1007/s40010-013-0066-8

Rajagopal, K.R., Gupta, A.S., 1984, "An Exact Solution for the Flow of a Non-Newtonian Fluid Past an Infinite Porous Plate,” ActaMechanica. 19, 158-161.

https://doi.org/10.1007/bf01560464

Samria, N.K., Prasad, R., Reddy, M.U.S., 1991, "MHD Free-Convection Flow of a Visco-Elastic Fluid Past an Infinite Vertical Plate," Astrophysics and Space Science.181, 135-145. https://doi.org/10.1007/bf00644120

Nabil, T.M., Eldabe, Sallam N. Sallam, Mohamed Y., Abou-zeid., 2012, "Numerical Study of Viscous Dissipation Effect on Free Convection Heat and Mass Transfer of MHD Non-Newtonian Fluid Flow Through a
Porous Medium,” Journal of Egyptian Mathematical society. 20, 139151.

https://doi.org/10.1016/j.joems.2012.08.013

Ramesh Chand, G. C., Rana., 2014, "Double Diffusive Convection in a Layer of Maxwell Viscoelastic Fluid in Porous Medium in the Presence of Soret and Dufour Effects,” Journal of Fluids, 1-7. https://doi.org/10.1155/2014/479107

Rashidi, M.M., Ali, M., Freidoonimehr, N., Rostami, B., Hossain, M.A.,2014, "Mixed Convective Heat Transfer for MHD Viscoelastic Fluid Flow Over a Porous Wedge With Thermal Radiation,” Advances in mechanical Eng. Volume 6.

https://doi.org/10.1155/2014/735939

Jha, A.K., Choudhary, K., Sharma, A., 2014,"Influence of Soret Effect on MHD Mixed Convection Flow of Visco-Elastic Fluid Past a Vertical Surface With Hall Effect,” Int. J. of Applied Mechanics and Engineering volume 19 issue 1.

https://doi.org/10.2478/ijame-2014-0007

Umamaheswar, M., Varma, S.V.K., Raju, M.C., 2013, “ Unsteady MHD Free Convective Visco-Elastic Fluid Flow Bounded by an Infinite Inclined Porous Plate in the Presence of Heat Source, Viscous Dissipation and Ohmic Heat," International Journal of Advanced Science and Technology, volume 61, $39 \quad-\quad 52$. https://doi.org/10.14257/ijast.2013.61.05

Attia, H.A., 2013, "Unsteady Flow of a Non-Newtonian Fluid Above a Rotating Disk With Heat Transfer," Int. J. of Heat and Mass Transfer volume 46 issue 14, 2695 - 2700.

https://doi.org/10.1016/s0017-9310 (03)00029-2

Prakash, J., Vijaya Kumar, A.G., Madhavi, M., Varma, S.V.K., 2014, "Effects of Chemical Reaction and Radiation Absorption On MHD Flow of Dusty Viscoelastic Fluid,” An International Journal of (AAM). (9)141156.

Ravikumar, V., Raju, M.C.,Raju, G.S.S., 2015,“Theoretical Investigation of an UnsteadyMHD Free Convection Heat and Mass Transfer Flow of a Non-Newtonian Fluid Flow Past a Permeable Moving Vertical Plate in the Presence of Thermal Diffusion and Heat Sink," Int. $J$. of Engineering Research in Africa volume 1690 - 109. https://doi.org/10.4028/www.scientific.net/jera.16.90

Kumar, B.R., Sravan Kumar, T., and Vijaya Kumar, A G., 2015 "Thermal Diffusion and Radiation Effects on Unsteady Free Convection Flow In the Presence of Magnetic Field Fixed Relative to the Fluid or the Plate”. Frontiers Heat and Mass Transfer, 6, 12, pp. 1-9. http://dx.doi.org/10.5098/hmt.6.12 